\title{
Immunity against hepatitis $B$ in a selected child population in Gampaha district, Sri Lanka, ten years after vaccination at two, four and six months of age
}

\author{
PJ Perera ${ }^{1}$, MD Fernando ${ }^{2}$, M Hapugoda $^{2}$, L Udayanga $^{2}$ \\ Sri Lankan Journal of Infectious Diseases 2022 Vol.12(1):E4 1-8 \\ DOI: http://dx.doi.org/10.4038/sljid.v12i1.8419
}

\begin{abstract}
Background: Hepatitis B (HB) infections are a global public health concern, which carry high morbidity and mortality. Immunization is the safe and effective intervention to prevent transmission. Currently, the Hepatitis B vaccine is given to children at two, four and six months of age in Sri Lanka. However, the capability of this regimen in providing long term protection up to adolescence and beyond remains a concern. The current study was conducted to assess the HB immune status of children, ten years after vaccination during infancy.
\end{abstract}

Methods: Antibody status of 150 children who received HB vaccine at two, four and six months of age was assessed 10 years after the last dose of vaccination. Some of these children had received liquid monovalent HB vaccine, while others were vaccinated with the Hexavalent vaccine. Children with HB antibody levels below the protective level were given a booster dose of monovalent recombinant HB vaccine, and antibody levels were reassessed in one month.

Results: At the initial assessment, 128 children (85\%) had protective levels of antibodies. Type of vaccine and gender had no significant effect on the prevalence of HB antibody levels $(p>0.05)$. All children who received the booster dose developed protective levels of antibodies.

Conclusions: Most of the children immunized against HB during infancy maintain protective antibody levels at 10 years of age. Children with antibody levels below protective levels produced a rapid anamnestic response following a booster dose.

Keywords: Hepatitis B, Immunity, Immunization, Antibodies

\section{Introduction}

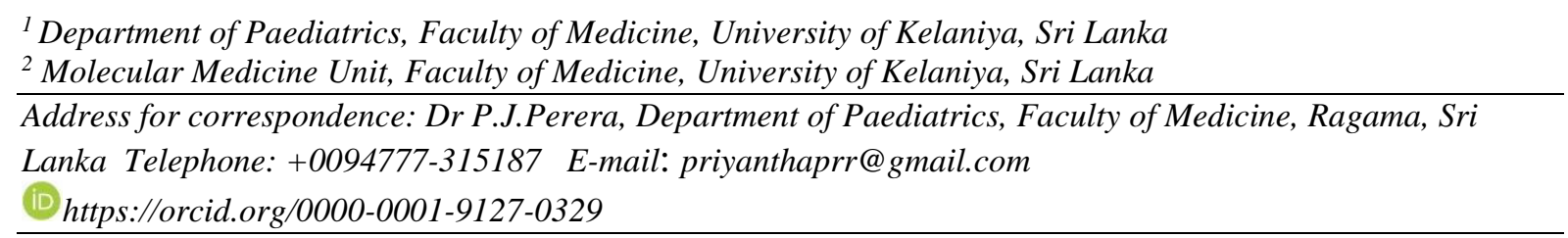

Received 5 October 2021 and revised version accepted 8 January 2022. Published on 25.2.22 
Hepatitis B (HB) infections are a global public health concern. Of the world population, around 248 million individuals $(3.6 \%)$ are positive for hepatitis B surface antigen. ${ }^{1}$ Approximately 686,000 died worldwide from complications related to acute or chronic HB infections in $2013 .^{2}$ Thus, inducing immunity against HB through immunization is a vital public health intervention.

Hepatitis B is hyper-endemic in Western Pacific and South East Asian regions, with carrier rates ranging from 5-35\%. ${ }^{3}$ In developed countries like North America, Australia and New Zealand, it is less than $1 \% .{ }^{4}$ Although located in a hyper-endemic region, Sri Lanka remains a low endemic country for Hepatitis B, with an estimated prevalence of less than $2 \% .^{5}$

Transmission of HB virus (HBV) mainly occurs through direct contact with blood or body fluids of an infected person, unprotected sexual contacts and vertical transmission from mother to child. Since HBV is capable of remaining infective outside the body for at least seven days, horizontal transmission through close interpersonal contact is possible. ${ }^{6,7}$ In low prevalent countries, HBV transmission is mainly during young adulthood through unprotected sexual activities.

Immunization remains the most effective method of preventing HBV transmission. In 1992, the World Health Organization recommended all countries to incorporate HB vaccination into their national childhood immunization programs. The present global hepatitis B vaccine coverage for 3 doses is estimated at $82 \% .^{8}$ The majority of Sri Lankan children are vaccinated at government immunization clinics, while a relatively small percentage are vaccinated in the private sector. In Sri Lanka, vaccination against HB was initiated in 2003 as a liquid monovalent vaccine, the vaccine was administered at two, four and six months of age, and no further booster doses were administered. ${ }^{9}$ Since 2008 , HB vaccine is administered along with four other vaccines as the pentavalent vaccine.$^{10}$ Children receiving vaccination from the private sector receive the $\mathrm{HB}$ vaccine as a component of the hexavalent vaccines.

For this vaccination strategy to be effective, immunity produced by the vaccination during infancy should last until adolescence and beyond. Even after many years of HB vaccination, there is still debate over the duration of long term protection. Requirement of a booster dose for long term protection is also debated. ${ }^{11}$ The minimal protective titre of HB antibodies is considered as $10 \mathrm{IU} / \mathrm{L}$, which is reliably measured with ELISA techniques. ${ }^{12}$

Long-term follow up studies are required to identify the protection provided by the HB vaccine. The incidence of HB infection among immunized cohorts and anamnestic response on exposure to HBs Ag can be used as evidence of protection. ${ }^{11}$ Several studies have shown a primary course of hepatitis B vaccination would provide protection against HB for more than 20 years, even with antibody levels that have fallen below protective levels. ${ }^{13-18}$ However, there are no studies conducted in Sri Lanka which have specifically assessed long term protection following HB immunization with recombinant HB vaccine given at two, four and six months. The current study was conducted to fill this gap in knowledge. We studied the immune status of children 10 years after vaccination with the recombinant $\mathrm{HB}$ vaccine, administered either as the liquid monovalent vaccine or as the hexavalent vaccine during infancy. 


\section{Methods}

The study was conducted at North Colombo Teaching hospital, Hemas hospital Wattala, Leesons hospital, Ragama and Faculty of Medicine, University of Kelaniya. As blood samples were required to check antibody levels, children brought for treatment at the above hospitals and in need of venesection were considered for recruitment. We therefore prevented any child being subjected to pain solely for the study purpose. Children with any chronic disorders that would interfere with the immune response to vaccination were excluded.

Children with documentary evidence of receiving three doses of HB vaccine at two, four and six months, with the last dose given 10 years ago were considered for the study. None of the children had past or present evidence of liver disease or any significant exposure putting them at risk of acquiring hepatitis $\mathrm{B}$ infection. The objectives and conduct of the study were explained to both the guardian and the child. Written consent from the mother and assent from the child were obtained before recruitment. Approximately $1 \mathrm{ml}$ of blood was collected in a plain bottle in addition to the blood collected for the patient's routine investigations. Serum was separated immediately and stored at $-20^{\circ} \mathrm{C}$.

According to Pierre et.al. sero-conversion rate following HB vaccination is around $90 \% .{ }^{11}$ Accordingly, a sample size of 150 was considered appropriate for the study using the EpiTools sample size calculator ${ }^{19}$, leaving an allowance for dropouts. Antibodies to the HB surface antigen (HBS-Ag) were quantitatively assessed using Monolisa ${ }^{\circledR}$ Anti-HBs PLUS ELISA kit (Bio-Rad-France), under direct supervision of trained and well experienced personnel. The manufacturer's instructions were followed, and all samples done in a single run.

Parents were informed about the immune status of their children. A booster dose of HB vaccine (Negeri B [GSK biological s.a.Rixesart-Belgium]) was offered free of charge to children with $\mathrm{HB}$ antibody titres less than $10 \mathrm{mIU} / \mathrm{L}$. All these children presented for the booster dose. The antibody titres were retested one month following the booster dose, employing the same procedure.

\section{Statistical analysis}

Geometric Mean Titre (GMT) for HB antibody levels in children were calculated and the Chi-square test of independence was used to investigate the association of gender and type of the vaccine (monovalent or hexavalent) on the protective levels of HB antibodies in children. In addition, independent sample t-test was used to assess the significance between the mean antibody levels of children vaccinated with hexavalent and monovalent vaccines 10 years after vaccination. IMB SPSS version 23 for windows 10 (IBM SPSS version 23 for windows $10)$, was used for the data analysis.

Ethical approval to conduct the study was obtained from Ethics Review Committee of the Faculty of Medicine, University of Kelaniya. Permission was obtained from respective hospital authorities. Participation in the study was voluntary. Written consent from the guardians were obtained along with the assent from the child. Parent or child had the right to withdraw from the study at any stage without facing any consequences. Confidentiality of the acquired data was maintained throughout the study. Serum samples remaining after antibody assay were discarded. 


\section{Results}

Out of 150 children recruited to the study, 68 were males $(45.3 \%)$, and $82(54.6 \%)$ were females. Monovalent HB vaccine had been received by $95(63.3 \%)$ children, while 55 $(36.7 \%)$ had received hexavalent vaccine. None of the recruited children had any significant exposure of acquiring HB infection. None had clinical evidence, past history or family history of

hepatitis.

Table 1. Antibody levels 10 years after Hep B primary vaccination

\begin{tabular}{|c|c|c|c|c|}
\hline $\begin{array}{c}\text { HB antibody } \\
\text { concentration (mIU/ml) }\end{array}$ & \multicolumn{2}{|c|}{ Number of children } & \multicolumn{2}{c|}{ Geometric Mean Titre } \\
\hline & $\mathrm{N}$ & $\%$ & GMT & $95 \%$ CI (mIU/ml) \\
\hline $0-10$ & 22 & 15 & 4.52 & $3.14-8.49$ \\
\hline$>10-100$ & 71 & 47 & 83.18 & $78.89-87.10$ \\
\hline$>100-1000$ & 42 & 28 & 407.38 & $334.20-493.17$ \\
\hline$>1000$ & 15 & 10 & 1047.13 & $1037.29-1067.89$ \\
\hline Total & 150 & 100 & & \\
\hline
\end{tabular}

Distribution of the antibody concentrations among the studied children, quantified based on the positive control series provided by the manufacturer is shown in Table 1, along with the Geometric Mean Titers. Based on this assay, 128 (85\%) children had HB antibody levels more than $10 \mathrm{mIU} / \mathrm{ml}$. The majority of the children had antibody concentrations between 10 $100 \mathrm{mIU} / \mathrm{ml}$, and 10\% had antibody concentrations above $1000 \mathrm{mIU} / \mathrm{ml}$.

Percentages of males and females having protective levels of antibodies were $85.3 \%(58 / 68)$ and $85.4 \%$ (70/82) respectively (Figure 1), showing no association of gender on long term protection $(\mathrm{p}>0.05)$.

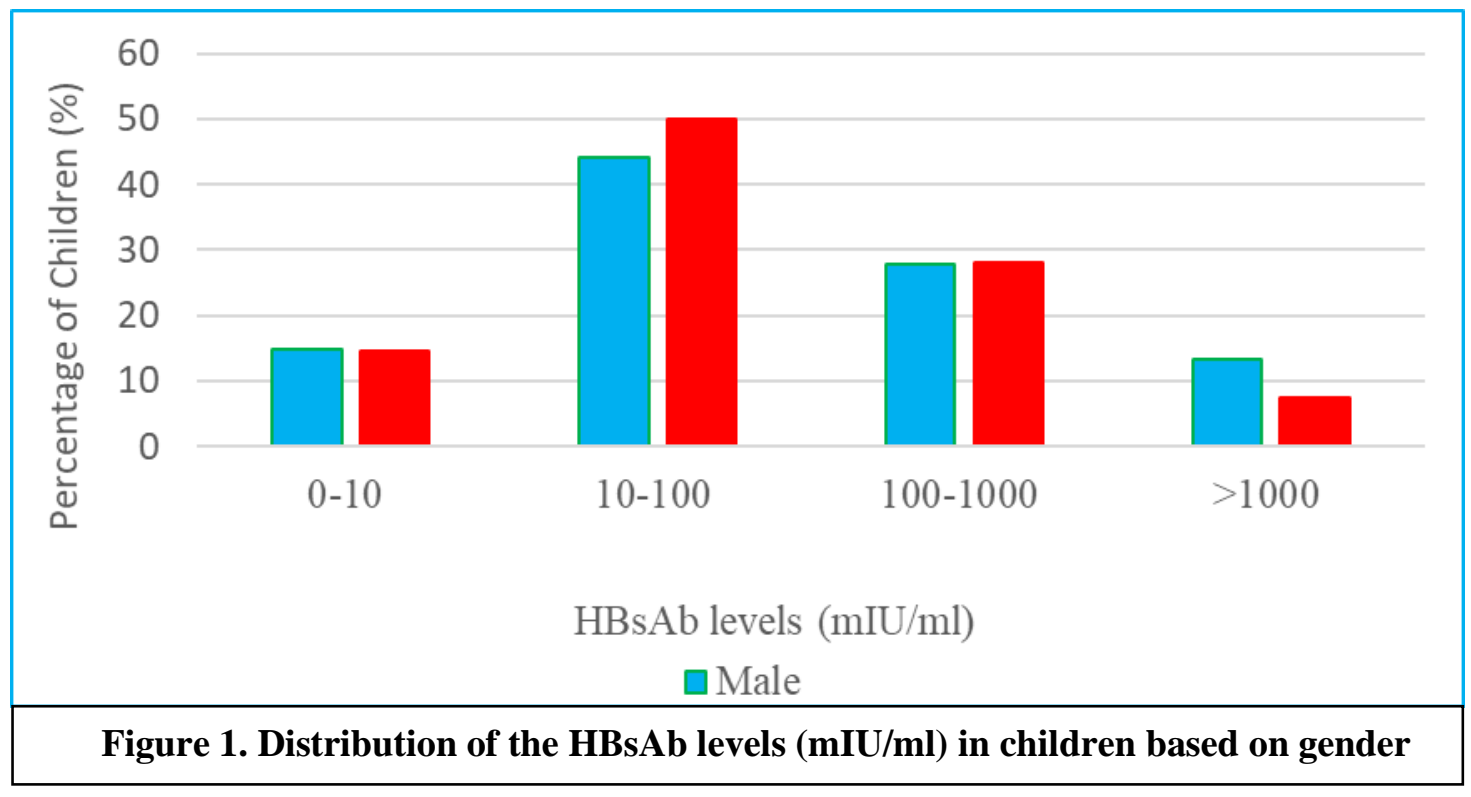

According to the type of the vaccine received, $82 \%$ (78/95) of the children who had the monovalent vaccine and 90\% (50/55) who had the hexavalent vaccine had protective levels of HB antibodies. However, this difference was not statistically significant ( $p>0.05)$. The 
mean GMT of HB antibody levels of the children vaccinated with monovalent vaccine remained as $180.08(144.54-223.87) \mathrm{mIU} / \mathrm{ml}$, while hexavalent vaccine resulted in a mean GMT of $187.65(138.49-302.04) \mathrm{mIU} / \mathrm{ml}$, along with a GMT difference of $10.18(7.58-$ $15.78) \mathrm{mIU} / \mathrm{ml}$ among the hexavalent and monovalent vaccines 10 years after vaccination. The results of the independent-sample t-test showed that the mean HB antibody levels of children vaccinated with hexavalent $(283 \mathrm{mIU} / \mathrm{ml})$ and monovalent $(279 \mathrm{mIU} / \mathrm{ml})$ vaccines did not demonstrate a significant difference ( $\mathrm{p}>0.05$ at $5 \%$ level of significance) 10 years since vaccination.

As depicted in Table 2, all 22 revaccinated children developed HB antibody levels above the protective level of $10 \mathrm{mIU} / \mathrm{ml}$. The mean GMT of HB antibody levels after revaccination were relatively high in children vaccinated with monovalent vaccine; $514.47 \mathrm{mIU} / \mathrm{ml}(357.52$ - 648.59), compared to children vaccinated with hexavalent vaccine $(458.89 \mathrm{mIU} / \mathrm{ml}$ with 302.37 - 567.67 at $95 \%$ confidence levels). The GMT difference of 11.53 (7.41 - 15.14) $\mathrm{mIU} / \mathrm{ml}$ between the hexavalent and monovalent vaccines groups was not significant.

Table 2. Concentration of HB antibody among revaccinated children

\begin{tabular}{|c|c|c|c|c|}
\hline $\begin{array}{c}\text { Concentrations of } \\
\text { HB antibodies } \\
\text { (mIU/ml) }\end{array}$ & \multicolumn{2}{|c|}{$\begin{array}{c}\text { Number of } \\
\text { children }\end{array}$} & \multicolumn{2}{c|}{ Geometric Mean Titre } \\
\hline & $\mathbf{N}$ & $\%$ & $\begin{array}{c}\text { GMT } \\
\text { (mIU/ml) }\end{array}$ & $\mathbf{9 5 \%}$ CI \\
\hline $0-10$ & 00 & 0.0 & - & $55.72-97.58$ \\
\hline$>10-100$ & 04 & 18.2 & 75.86 & $887.16-979.49$ \\
\hline$>100-1000$ & 10 & 45.4 & 933.25 & $1023.29-1080.94$ \\
\hline$>1000$ & 8 & 36.4 & 1047.13 & \\
\hline Total & $\mathbf{2 2}$ & $\mathbf{1 0 0}$ & & \\
\hline
\end{tabular}

\section{Discussion}

Sri Lanka is a low prevalence country for HB, with a minimal risk of vertical and horizontal transmission. Health care providers in Sri Lanka take stringent precautions to prevent iatrogenic HB infections. All blood donors are routinely screened for HB with DNA PCR, and all interventional procedures are carried out under sterile conditions. Intravenous drug abuse is also rare in Sri Lanka. ${ }^{10}$ Thus, like other low prevalence countries, the most likely mode of $\mathrm{HB}$ transmission is unprotected sexual contacts during adolescence and later., ${ }^{6,7}$ Hence immunity against $\mathrm{HB}$ during adolescence and later is vital for prevention of $\mathrm{HB}$ transmission in a low prevalence country like Sri Lanka. In this context it is important to ascertain whether immunization carried out against Hep B during early infancy is long lasting, as transmission risk during childhood is minimal. ${ }^{13-16}$

According to our study, whether the $\mathrm{HB}$ vaccine is given as a monovalent vaccine or as a combined vaccine, the protection provided against $\mathrm{HB}$ at 10 years is not significantly different. The main advantage of administering HB vaccine through a combined vaccine is ensuring a higher acceptance as no additional prick for HB vaccine is required. ${ }^{10}$ The present coverage for three doses of HB vaccine in Sri Lanka is $98 \%{ }^{20}$ which is significantly higher than the global figure of $82 \% .^{8}$ The main reason for the high coverage is carrying out vaccinations alongside other child welfare clinic activities conducted all around the country. Acceptance and compliance of a $\mathrm{HB}$ vaccination program initiated around adolescence is 
likely to be low. The main advantages of the current vaccination schedule are fewer painful pricks to the child and clinic visits, reduced workload on health staff and improved cost effectiveness. Hence, the current schedule of two, four and six months remains the ideal, as long as it provides adequate protection till adolescence and beyond.

Debate over duration of protection provided by $\mathrm{HB}$ vaccine has continued over a long period. ${ }^{11}$ Some studies have shown that protection lasts beyond 20 years. ${ }^{13}$ Studies have also demonstrated that protection provided by $\mathrm{HB}$ vaccine outlast the duration of detectable protective antibody levels. ${ }^{21,22}$ In our study, $15 \%$ of children did not have protective antibody concentrations ten years after immunization. As we have not tested the immune response in these children following the course of primary immunization, it is reasonable to speculate that some of them may not have had a seroconversion at all. However, the sharp and rapid anamnestic rise of antibody levels following booster dose observed in these children suggest seroconversion following primary vaccination. This observation also confirms the findings of previous studies, which suggest immune memory to remain, even when the antibody levels have dropped below protective levels. ${ }^{11,17,18,23}$ However, these studies were either in adults or in children who had the first dose of the HB immunization during the latter part of childhood or at the time of birth. Some studies were conducted with plasma derived HB vaccine prior to availability of the recombinant vaccine. ${ }^{18}$ According to the world medical literature, our study is the first to investigate the long-term protection following a primary course of recombinant $\mathrm{HB}$ vaccination, administered at two, four and six months of age.

However, an issue that needs addressing is whether the anamnestic response is quick enough to prevent the disease. The longer incubation period of HB may be a favourable factor in this regard. The immune response is not only humoral and especially in viral infections, but $\mathrm{T}$ cell response also plays an important role in protection. ${ }^{15}$ Following up vaccinated individuals for clinical or serological evidence of HB infection is the ideal method of demonstrating longterm protection. None of the children included in our study had any clinical evidence of HB infection at present or in the past. However, due to limited resources we did not perform serological studies for HB infection amongst these children.

In contrast to our findings, two studies reported only $51 \%(18 / 35)$ of previously vaccinated individuals producing an anamnestic response following a booster dose, given 15 years after a primary course of HB vaccination. ${ }^{24}$ In both these studies, the first dose was given at birth. The lower anamnestic response documented by these two studies may be due to an immature immune system at birth and/or antibodies received either from mother or given to the baby, preventing an adequate immune response. Thus, unless there is a risk of vertical transmission, commencing $\mathrm{HB}$ vaccination at two months of age seems to ensure long term protection rather than commencing vaccination at birth.

\section{Conclusion}

It can be concluded that $\mathrm{HB}$ vaccine in the hexavalent form is equally effective as monovalent $\mathrm{HB}$ vaccine. With time, it is likely that $\mathrm{HB}$ antibody levels tend to fall below protective levels in some vaccinated individuals. However, as sharp anamnestic rise of antibodies was observed in these previously vaccinated individuals following a booster dose, it is likely that they will respond to a natural infection in a similar fashion. It is likely that a booster dose of $\mathrm{HB}$ vaccine is not indicated in individuals who received a successful primary course of $\mathrm{HB}$ vaccine at two, four and six months. 


\section{Limitations}

The main limitation of our study is the limited sample size of 22 during the second part of the study. Another limitation is that findings after 10 years of vaccination may not necessarily guarantee similar immune response several decades later. A definitive answer to the question of 'necessity of a booster dose' would be obtained only from a long term follow up study with a larger sample size.

\section{Declarations}

Acknowledgement : We thank all children for their participation and their parents for consenting to include their children in the study Funding: This research did not receive any specific grant from funding agencies in the public, commercial, or not-for-profit sectors.

Conflict of Interest: All authors have read and approved the manuscript. Authors declare there are no conflicts of interest in publishing this article.

Ethics statement : Ethical approval to conduct the study was obtained from the Ethics Review Committee of the Faculty of Medicine, University of Kelaniya (p22/03/2013). Permission was obtained from respective hospital authorities to conduct the study. Participation in the study was entirely voluntary. Written consents form the guardians and the assent from the child was obtained. Parent or the child had the right to withdraw from the study at any stage, without facing any consequences. The confidentiality of the acquired data was maintained throughout the study.

Serum samples remaining after antibody assay were discarded

Author contributions

PJP was involved in conceptualization, planning \& proposal writing, data analysis and writing the manuscript.

MDF was involved in planning \& proposal writing, sample collecting, laboratory analysis, data analysis and writing the manuscript.

MH was involved in conceptualization, planning \& proposal writing, laboratory analysis and writing the manuscript

LU was involved in laboratory analysis, data analysis and writing the manuscript.

\section{References}

1 Schweitzer A, Horn J, Mikolajczyk RT et al. Estimations of worldwide prevalence of chronic hepatitis B virus infection: a systematic review of data published between 1965 and 2013. Lancet. 2015; 386:1546-55. doi: http://dx.doi.org/10.1016/S0140-6736(15)61412-X

2 McGlynn KA, Petrick JL, London WT. Global epidemiology of hepatocellular carcinoma: an emphasis on demographic and regional variability. Clin Liver Dis. 2015; 19:223-38.

doi: http://dx,doi.org/10.1016/j.cld.2015.01.001

3 Gust I D. Epidemiology of hepatitis B infection in the Western Pacific and South East Asia. Gut. 1996; 38(Suppl 2):S18-S23. doi: http://doi.org/10.1159/000050066

4 Chen CJ, Wang LY, Yu MW. Epidemiology of hepatitis B virus infection in the Asia-Pacific region. J Gastroenterol Hepatol. 2000; 15:Suppl:E3-6.

doi: http://doi.org/ doi: 10.1046/j.1440-1746.2000.02124.x. PMID: 10921373

5 Noordeen F, Pitchai FNN, Rafeek RA. A review of hepatitis B virus infection in Sri Lanka. Sri Lankan Journal of Infectious Diseases, 2015;5(2):42-50.

doi: http://dx.doi.org/10.4038/ sljid.v5i2.8087

6 Bond WW, Favero MS, Petersen NJ et al. Survival of hepatitis B virus after drying and storage for one week. Lancet. 1981; 317(8219):550-551.

doi: http://dx.doi.org/10.1016/S0140-6736(81)92877-4

7 Davis LG, Weber DJ, Lemon SM et al. Horizontal transmission of hepatitis B virus. Lancet. 1989; 1(8643):889-93. doi: https://doi.org/10.1016/S0140-6736(89)92876-6

8 World Health Organization. Immunization coverage. Fact sheet No. 378. Updated September 2015. Available at http://www.who.int/mediacentrelfactsheets/fs378/en/.

9 Ministry of health, Sri Lanka. Child health development record 2016.

10 Epidemiology Unit, Ministry of Health Sri Lanka 2012. National Expanded Programme on Immunization, Sri Lanka. Immunization Handbook. Third Edition

11 Pierre Van Damme. Long-term Protection after Hepatitis B Vaccine. The Journal of Infectious Diseases. 2016; 214(1):1-3. doi: https://doi.org/10.1093/infdis/jiv750

12 Francis DP, Hadler SC, Thompson SE et al. The prevention of hepatitis B with vaccine: report of the Centres for Disease Control multi-centre efficacy trial among homosexual men. Ann Intern Med. 1982; 97(3):362-6. doi: http://dx.doi.org/10.7326/0003-4819-97-3-362 
13 Poovorawan Y, Chongsrisawat V, Theamboonlers A et al. Long-term anti-HBs antibody persistence following infant vaccination against hepatitis $\mathrm{B}$ and evaluation of anamnestic response: a 20-year follow-up study in Thailand. Hum Vaccin Immunother. 2013; 9(8):679-84. http//doi:10.4161/hv.24844. PMID:23732904.

14 Spada E, Romano L, Tosti M et al. Hepatitis B immunity in teenagers vaccinated as infants: an Italian 17-year follow-up study. Clin Microbiol Infect. 2014; 20(10):680-6. doi: http://dx.doi.org/10.1111/1469-0691.12591

15 Zhu C-L, Liu P, Chen T et al. Presence of immune memory and immunity to hepatitis B virus in adults after neonatal hepatitis B vaccination. Vaccine. 2011; 29(44):7835-41. doi http://dx.doi.org/10.1016/j.vaccine.2011.07.098

$16 \mathrm{Ni} \mathrm{YH,} \mathrm{Chang} \mathrm{MH,} \mathrm{Wu} \mathrm{JF} \mathrm{et} \mathrm{al.} \mathrm{Minimization} \mathrm{of} \mathrm{hepatitis} \mathrm{B} \mathrm{infection} \mathrm{by} \mathrm{a} 25$ year universal immunization program. J Hepatol. 2012; 57:730-5. doi:10.1016/j.jhep.2012.05.021

17 Mendy M, Peterson I, Hossin S et al. Observational study of vaccine efficacy 24 years after the start of hepatitis B vaccination in two Gambian villages: no need for a booster dose. Plosone. 2013; 8:e58029. doi: http://dx.doi.org/10.1371/journal.pone.0058029

18 Wang F, Shen L, Cui F et al. The long-term efficacy, 13-23 years, of a plasma-derived hepatitis $\mathrm{B}$ vaccine in highly endemic areas in China. Vaccine. 2015; 33:2704-9. doi: http://dx.doi.org/10.1016/j.vaccine.2015.03.064

19 Calculate sample size for freedom testing with imperfect tests. Available at: https://epitools.ausvet.com.au/freecalctwo. Last viewed 07.02.2022

20 Ministry of Health, Sri Lanka Annual Health Bulletin. 2016.

21 Bialek SR, Bower WA, Novak R et al. Persistence of protection against hepatitis B virus infection among adolescents vaccinated with recombinant hepatitis B vaccine beginning at birth: a 15-year follow-up study. Pediatr Infect Dis J. 2008; 27(10):881-5. doi: 10.1097/INF.0b013e31817702ba.

22 Leuridan E, Van Damme P. Hepatitis B and the need for a booster dose, Clinical Infectious Diseases, 2011; 53(1):68-75. doi: https://doi.org/10.1093/cid/cir270

23 Bruce MG, Bruden D, Hurlburt D et al. Antibody levels and protection after Hepatitis B vaccine: results of a 30 year follow up study and response to a booster dose. The Journal of infectious disease. 2016; 214:16-22. doi: http://dx.doi.org/10.1093/infdis/jiv748

24 Hammitt LL, Hennessy TW, Fiore AE et al. Hepatitis B immunity in children vaccinated with recombinant hepatitis B vaccine beginning at birth: a follow-up study at 15 years. Vaccine. 2007; 25(39-40):6958-64. doi: http://dx.doi.org/10.1016/j.vaccine.2007.06.059 